\title{
Lænnec und sein Vorlesungsmanuskript von 1822
}

\author{
Von Erwin H.ACKerknecht
}

René-ThÉophile-Hyacinthe Lannec ist wohl der größte Vertreter jener revolutionären «Spitalmedizin», durch die zwischen 1800 und 1850 Paris das Mekka der Weltmedizin wurde, jener Schule, welche im medizinischen Denken die Läsion an Stelle des Symptoms als Dominante setzte ${ }^{1}$ und so die großen Virtuosen der Diagnose hervorbrachte. Er ist typisch für sie in seinem Interesse an pathologischer Anatomie und physikalischen Untersuchungsmethoden, seinem Appell an die Statistik und in seiner Tendenz zur therapeutischen Skepsis ${ }^{2}$ und zur Spezialisierung.

Es ist bedeutsam, daß er, 1781 in Quimper geboren ${ }^{3}$ Bretone war wie andere Frondeure seiner Zeit, z. B. Lamennais, Chateaubriand oder sein zu seinen Lebzeiten siegreicher Konkurrenz Broussais ${ }^{4}$. Es war auch folgenschwer, daß er (unter Obhut seines Onkels Guillaume in Nantes) mit 14 $1 \frac{1}{2}$ Jahren seine medizinische Ausbildung als Militärchirurg 3. Klasse begann. $\mathrm{Er}^{5}$ und BICHAT ${ }^{6}$ haben mit Recht betont, daß die pathologische Anatomie, die Grundlage der neuen Orientierung, von den Chirurgen in die Medizin gebracht worden ist ${ }^{\text {? }}$.

1 E.H.Ackerknecht, La médecine à Paris entre 1800 et 1850, Paris 1958. Siehe auch A. Corleu, Le Centennaire de la Faculté de médecine à Paris, Paris 1896. P.Delaunay, D’une révolution à l'autre, 1789-1848, Paris 1949. P.Astruc, La médecine au XIX siècle, Progrès Med. 1957, Nos 15-18; 1958, $\mathrm{N}^{\mathrm{os}}$ 1-4. M.Foucault, Naissance de la clinique, Paris 1963.

2 E.H.Ackerknecht, Die Therapie der Pariser Kliniker zwischen 1795 und 184.0, Gesnerus 15 (1958) 151. W. ARtelt, Louis' amerikanische Schüler und die Krise der Therapie, Sudhoffs Arch. 42 (1958) 291.

3 Zur Biographie Lænnecs sind noch immer unübertroffen: A. RouxeAu, Lænnec avant 1806, Paris 1912; id., Lannec après 1806, Paris 1920.

4 E. H.Ackerknecht, Broussais, Bull.Hist.Med.27 (1953) 320.

5 Manuskript, Vorlesung «Pathologische Anatomie » 1803.

6 F.X.Bichat, Pathologische Anatomie, Leipzig 1827, S. 5.

7 O. Temkin, The Role of Surgery in the Rise of modern Medical Thought, Bull. Hist. Med. 25 (1951) 248. E.H.Ackerknecht, Pariser Chirurgie von 1794 bis 1850, Gesnerus 17 (1960) 137. 
Mit 20 Jahren kam er schließlich als Medizinstudent nach Paris und wurde ausschlaggebend von vier Männern beeinflußt: Corvisart, Bichat ${ }^{8}$, G.L.BAyle und Dupuytren. Auf Corvisart geht zurück seine Neigung zur pathologischen Anatomie, zur Perkussion, zu den Krankheiten des Brustraums, zum therapeutischen Skeptizismus, sein - trotz aller Neuerungen, die er brachte - prononcierter Traditionalismus (Corvisart unterrichtete bekanntlich nach Stoll, dessen Buch ein Kommentar zu BoerhaAve und noch durchaus im Geist des 5.Jahrhunderts v. Chr. abgefaßt war). Bichat, Bayle und Dupytren lehrten ihn ebenfalls pathologische Anatomie. Bayle außerdem die Anfänge der Auskultation, die Grundlagen der Phthisen- und Tumorenlehre - und den Royalismus jesuitischer Färbung ${ }^{9}$.

Die erste Arbeit Lænnecs, die aufhorchen ließ, war sein 1802 im Journal de Médecine, Chirurgie et Pharmacie erschienener Artikel über mehrere Fälle von Peritonitis. Der nicht genügend anerkannten Entzündung einer serösen Membran geweiht, ist er ganz im Geiste Bichats gehalten. Derselbe Jahrgang des «Journals» enthält auch einige kleinere anatomische Entdeckungen Lænnecs. Bereits im nächsten Jahr löste sich der Zweiundzwanzigjährige von Dupuytren, dessen Gehilfe er gewesen war, gab seinen eigenen Kurs in pathologischer Anatomie und kündigte ein Lehrbuch über dieses Gebiet an, das ebensowenig erschienen ist ${ }^{10}$ wie ein ebenfalls angekündigtes Buch seines Ex-Chefs, der sich nun hauptamtlich der Chirurgie zuwandte.

Auch Lænnec, der subjektiv und objektiv vor allem pathologischer Anatom war, mußte nun aus ökonomischen Gründen 1804 in die Privatpraxis. Als einer der größten Kliniker aller Zeiten, hat er diese Laufbahn also eindeutig wider Willen ergriffen. Dies hat ihn nicht gehindert, sowohl wissenschaftlich Außerordentliches zu leisten als auch ein hochgeschätzter Praktiker zu werden. Napoleons Onkel, Kardinal Fesch, Chateaubriand, dessen Frau, Lamennais oder Madame de Staël waren z.B. seine Patienten.

8 Diesem weihte Lænnec einen wahren Kult, obwohl er ihm in vielem, z.B. seinem besonderen Vitalismus, nicht folgte. Während Lænnec im allgemeinen in seinen Manuskripten auch Eigennamen klein schreibt, ist der von Bichat stets groß geschrieben!

9 Über G.L.Bayle s. A.L. Bayle et Thillaye, Biographies Médicales, Paris 1855, Band II, S. 884 .

10 Über die Notizen zu dieser Vorlesung bzw. Buch s. Fußnoten 21 und 22. Über Dupuytren s. L.Delhoume, Dupuytren, Paris 1935. Es ist bezeichnend, daß Cuvier (Rapport historique sur les progrès des sciences naturelles, Paris 1810, S. 346) als führende pathologische Anatomen der Periode Portal, Bichat, Dupuytren, Corvisart, Bayle und LÆNNEC nennt. 
1816 wurde er endlich Arzt am Hôpital Necker, d.h. er erhielt wieder die Möglichkeit, wissenschaftlich zu arbeiten. Im selben Jahr erfand er das Stethoskop und begann die Abfassung seines unsterblichen Traité de l'auscultation médiate et des maladies des poumons et du cœur. Nach Erscheinen desselben (1819) war seine Gesundheit bereits so erschüttert - er litt an Lungentuberkulose -, daß er sich bis 1822 auf sein Landgut Kerlouarnec zurückziehen mußte. Nach seiner Rückkehr nach Paris wurde er - wegen seiner legitimistisch-jesuitischen Auffassungen bei Hof ebenso beliebt wie bei Kollegen und Studenten unbeliebt ${ }^{11}$ - Professor am Collège de France, Professor für klinische Medizin der Charité und Leibarzt der Kronprinzessin. Dieser enormen Belastung, zu der noch der Kampf mit Broussais hinzutrat, war er physisch nicht gewachsen. Bereits 1826 ist er, $45 \mathrm{Jahre}$ alt, kurz nach Vollendung der 2. Auflage seines «Traité» in Kerlouarnec gestorben. Er hat eine Reihe bedeutender ausländischer Schüler gehabt wie Hodgkin, S.G.Morton, James Clark, Fossati, Retzius usw. Der Erfinder der Auskultation und des Stethoskops war nicht nur ein begabter Musiker und Drechsler, er trat auch als Dichter, Philologe, politischer Pamphletschreiber und Zeichner hervor.

Lænnecs Doktorarbeit aus dem Jahr 1804, Propositions sur la doctrine d'Hippocrate relativement à la médecine pratique, beschäftigt sich überraschenderweise nicht mit pathologischer Anatomie, sondern mit der medizinischen Tradition, als deren profunder Kenner und Verehrer er sich erweist. Er sieht sehr gut den Unterschied zwischen Hippokrates, der vor allem an den «symptomes communs» der Krankheiten und der Prognose, und den Ärzten seiner Generation, die in erster Linie an Diagnose und den «symptomes propres» interessiert sind. Paradoxerweise erwägt der zukünftige Entdecker so zahlreicher «symptomes propres», ob man nicht zu Hippokrates zurückkehren müsse ${ }^{12}$. Er schließt mit einem Zitat von KLEIN : «Liberam profiteor medicinam, nec ab antiquis sum nec ab novis; utrosque ubi veritatem colunt sequor; magni facio saepius repetitam experientiam.»Es ist in diesem Zusammenhang bezeichnend, daß er von 1805 bis 1814 jährlich über die «epidemische Konstitution» berichtete.

11 Es bleibt allerdings unklar, warum RÉCAMIER, Esquirol oder CRUveiLHIER trotz denselben Auffassungen nicht dieselbe Unbeliebtheit erfuhren.

12 l.c., S. 31. 
Auf dem Gebiet der pathologischen Anatomie hat er trotz intensiver Betätigung ${ }^{13}$ nur einige Zeitschriftenartikel und Beiträge zum Dictionnaire des Sciences Médicales veröffentlicht. Am wichtigsten von diesen ist wohl sein Artikel "Anatomie pathologique» im Dictionnaire des Sciences Médicales, Band 2, S. 46-61, Paris $1812^{14}$. Er bezeichnet die pathologische Anatomie dort als die «Fackel der Medizin» und möchte sie als selbständige Disziplin etablieren. Zu diesem Zweck bietet er jene an Bichat anschließende, aber über ihn hinausgehende pathologisch-anatomische Klassifikation, die er schon 1803 in seinem Kurs gelehrt hatte und über die es zu dem für ihn siegreichen Prioritätsstreit mit Dupuytren gekommen war. Die «maladies organiques» (nur für sie ist die pathologische Anatomie zuständig - die «maladies nerveuses» hinterlassen ja keine Läsionen) sind in vier Untergruppen geteilt, von denen die vierte: «altérations de texture», wieder in vier Untergruppen zerfällt. Deren letzte, die «tissus accidentels» teilt Lænnec nun ein in «analogues», d.h. Gewebe, die Parallelen im Normalen haben, und «non-analogues», solche, die keine derartige Parallelen haben. Das ist neu, von grundlegender Bedeutung für die Krebsforschung und wird, nachdem sich die Morphologen jahrzehntelang über die Existenz von analogielosen Geweben gestritten haben ${ }^{15}$, im 20. Jahrhundert wieder ein Zankapfel für die Biochemiker. An «nonanalogues» beschreibt Lænnec die «tubercules», den «squirrhe» und die von ihm abgesonderten Krebsformen «encephaloide» und «mélanoses». Sie alle haben eine Tendenz zur fortwährenden Veränderung und beeinflussen das Gesamtbefinden.

Lænnecs großer Wurf war sein 1819 erstmalig erschienener zweibändiger Traité de l'auscultation médiate et des maladies des poumons et du cœur (wir zitieren im folgenden aus der besser aufgebauten 2. Auflage von 1826). Während die Größe anderer Kliniker (z.B. BoerhaAve, Schönleiv) sich nicht in ihren Schriften spiegelt, war es Lænnec vergönnt, sich in diesem Buch adäquat auszudrücken. Als sein Ziel bezeichnet er im Vorwort (S. XXV): 1. am Leichnam einen pathologischen Fall auf Grund physikalischer Organveränderungen zu unterscheiden; 2. denselben Fall beim

13 Aus seinen Manuskripten geht z.B. hervor, daß er allein im September und Oktober 181622 Sektionen vornahm.

14 Seine Beiträge zum «Dictionnaire» sind alle Abkömmlinge seiner Vorlesung von 1803 und seines berühmten Mémoires über Würmer von 1804. Es handelt sich um die Artikel: Ascaride, Cartilage accidentel, Crinon, Cucurbitain, Dégéneration, Désorganisation, Dytrachyeros, Encephaloide, Filaire, Fibreux accidentel, Fibrocartilage.

15 E.H.Ackerknecht, Rudolf Virchow, Stuttgart 1957, S. 84. 
Lebenden wiedererkennen an gewissen Zeichen, die möglichst physikalischer Natur sein sollten und unabhängig von Symptomen, d.h. den veränderlichen Störungen der Lebenstätigkeit, welche sie begleiten; 3. die Krankheit mit denjenigen Mitteln, welche die Erfahrung als die wirksamsten erwiesen hat, behandeln. Auch wenn er es nicht bereits in der lateinischen Widmung an die Pariser Fakultät sagen würde, ist es danach klar, daß er gegen Systeme ist, besonders gegen das gerade herrschende «ätiologische» von Broussais, der alle Krankheiten aus der Gastreonteritis erklärt. Er lehnt auch Pinels nosologische Bemühungen ab (I, 133), nicht aber dessen essentielle Fieber. Er betrachtet Theorien überhaupt nur als Gedächtnishilfen, «Gerüste» (I, 183), In seiner Theoriefeindlichkeit ${ }^{16}$ geht er sogar so weit, daß er, wie übrigens schon Bichat, auch die Mikroskopie ablehnt (I, 280).

Die ersten 130 Seiten sind dann den Untersuchungsmethoden des Brustraumes gewidmet, unter denen natürlich die von ihm erfundene mittelbare Auskultation vermittels des Stethoskops die Hauptrolle spielt. Eine Diskussion der hier geschilderten Phänomene scheint überflüssig, da sie noch heute jedem Medizinstudenten eingehämmert werden. Unter denen, die er als Zeugen für die Richtigkeit seiner Angaben nennt, befindet sich immer wieder sein Freund RÉCAMIER ${ }^{17}$.

Unter den Krankheiten der Bronchien finden wir die von ihm gefundenen Bronchektasien, unter denen der Lunge das von ihm zuerst beschriebene vesikuläre Emphysem. Bei der Lungenentzündung gab Lænnec Brechweinstein in hohen Dosen, den er auch bei Meningitis, Polyarthritis, Chorea, Phlebitis, Ophthalmie und Anginen empfahl. Er hatte diese Methode von RASORI übernommen, ohne sich dessen Theorien zu eigen zu machen (I, 494). Dies war seine Haltung auch bei anderen Drogen (z. B. I, 182). Er glaubte statistisch nachweisen zu können, daß er die Pneumoniemortalität so von 1 in 6 auf 1 in 28 reduziert habe (I, 501). Seine Antimontherapie war einer der Punkte, an dem man Broussais' Kritik am ehesten

16 id., Elisha Bartlett and the philosophy of the Paris Clinical School, Bull. Hist. Med. 24 (1950) 43. Zur Philosophie der damaligen Klinik s. auch: G. Rosen, The Philosophy of Ideology and the Emergence of Modern Medicine, Bull. Hist. Med. 20 (1946) 328. O.Temkin, The Philosophical Background of Magendie's Physiology, ibid. 20 (1946) 10. E.Lesky, Cabanis und die Gewißheit der Heilkunst, Gesnerus 11 (1954) 152. P. LaIN Entralgo, Bichats Sensualism and Vitalism, J. Hist. Med. 3 (1948) 4.

17 P.Trialre, Récamier, Paris 1899; im Vaterland des Propheten ging man übrigens bald zur unmittelbaren Auskultation über. 
billigen kann. Sie überrascht in ihrer Gewaltsamkeit, da er sonst sehr viel von der Heilkraft der Natur hielt (schon Neuburger hat in seinem Buch über diesen Gegenstand eindrücklich darauf hingewiesen) und therapeutisch häufig skeptisch-zurückhaltend blieb.

Den Höhepunkt des Buches stellen die 200 Seiten füllenden makroskopischen pathologisch-anatomischen und auskultatorischen Beschreibungen der Lungentuberkulose dar, denen seither wenig hinzugefügt worden ist. Hier ist er weit über seinen Freund Bayle hinausgekommen. Was für diesen noch verschiedene Arten der Phthise waren, sind ihm Stufen eines Prozesses. In diesen Kapiteln wird Lænnec auch zum großen Unitarier der Tuberkulose, indem er auf ihr Vorkommen in verschiedenen Formen in allen Organen ( $I, 555)$, übrigens häufig im Latenzstadium (I, 699), hinweist. Der Arzt kann sie noch nicht heilen, wohl aber die Natur (II, 769). Die Ursachen sind ungewiß. Er lehnt die Charakterisierung der Phthise als chronische Lungenentzündung durch Broussais ab. Am besten gesichert als Ursachen sind die «passions tristes», die auch am meisten zur Entstehung anderer Neubildungen, wie dem Krebs, beitragen (I, 6461). Die Psychosomatik ist wirklich nicht so neu, wie sie manchmal denkt ${ }^{18}$. Tatsachen, die wir heute auf die von ihm geleugnete Kontagiosität zurückführen würden, interpretiert er so im psychologischen Sinne. Auch die Erblichkeit erkennt er als Ursache an ${ }^{19}$.

Lungentumoren hat er nur wenige gesehen. Entgegen einer pathologischanatomischen Zeitmode, die das spasmodische Asthma völlig zugunsten des Asthma cardiale aufgeben möchte, hält er an der Existenz desselben fest ${ }^{20}$. Nervöse Beschwerden behandelt er hier wie auch am Herzen häufig mit dem Magneten (II, 97). Unter den Pleuritiden beschreibt er als erster die hämorrhagische. Er tritt für die Operation des Empyems ein. In einer Fußnote zum Sektionsprotokoll einer Pleuritis mit Brustkorbschrumpfung (II, 196) schafft er den Begriff der Lebercirrhose. Auch den Pneumothorax beschreibt er als erster korrekt.

Seine Kapitel über Herzleiden sind, bis auf die neuen diagnostischen Zeichen, die er fand, weniger hervorragend. Er folgt hier weitgehend Corvisart und schlägt sich mit dem Broussais-Schüler Bouillaud herum, für den alles «endocardite» war.

18 D. Schneider, Die Psychosomatik von Pinel bis Trousseau, Zürich 1963.

19 H.Semadeni, Die Erbkrankheiten um 1850, Zürich 1960, S. 53.

20 Bei dieser Gelegenheit zitiert er übrigens ein Experiment aus JoH. MüLLERs Doktorarbeit (II, 93). 
Außer diesen Veröffentlichungen Lænnecs existieren noch sein Manuskript für seine pathologisch-anatomischen Vorlesungen von 1803 (Anzahl der Vorlesungen nicht mehr feststellbar, etwa $100 \mathrm{~S}$.) ${ }^{21}$ und dasjenige für seinen zweijährigen «Cours de médecine» am Collège de France, der das Gesamtgebiet der Medizin umfaßte und den er zweimal (1822-24 und 1824-26) abhielt. Das letztere Manuskript umfaßt 1600 Seiten und 161 Vorlesungen ${ }^{22}$. Beide sind nur in Stichworten abgefaßt und teilweise (wohl beim zweiten Abhalten des Kurses) intensiv überarbeitet.

Das pathologisch-anatomische Manuskript von 1803 bringt nicht viel Neues. Die Einführung entspricht fast wörtlich dem Dictionnaire-Artikel von 1812. Nur ist seit 1812 ein Angriff auf die «radikalen Empiriker» (wahrscheinlich war Dupytren gemeint, vielleicht auch Lieutaud) und ein Eintreten für pathologische Chemie weggeblieben. Eine Anweisung zur Autopsietechnik stützt sich auf Morgagni. Die Neubildungen und die Degenerationen werden relativ ausführlich behandelt. Von der 9. Vorlesung an wird die pathologische Anatomie der Gewebe und Systeme, beginnend mit den serösen Membranen, noch ganz im Sinne Bichats abgehandelt. Nur bis zum Knochensystem (24. Vorlesung) sind die Notizen halbwegs vollständig. Die Stichworte auf den letzten 40 Seiten des Manuskripts sind überaus fragmentarisch.

Die erste der Collège-de-France-Vorlesungen ist im Januar 1823 als erster Artikel in der ersten Nummer der berühmten Archives Générales de Médecine erschienen. Sie grenzt sich mit großer Schärfe sowohl von den reinen Empirikern wie von den sogenannten Ätiologen ab. Diese letztere Richtung wird durch die ganze Medizingeschichte verfolgt. Sie sei immer die Zuflucht der Denkfaulen gewesen. Besonders charakteristisch für sie seien das Leugnen unbequemer Tatsachen, Verachtung der genauen Beobachtung und des Hippokrates, ihr Größenwahn und ihr Spekulieren auf den Enthusiasmus der Jungen. Ihr Prototyp sei Paracelsus, hinter dessen Maske Broussais unschwer zu erkennen ist. Seine eigene Richtung bezeichnet Lænnec als die der «observation», die, gestützt auf Beobachtung und Nachdenken,

21 ConNIL hat die erste Hälfte dieses Manuskripts 1884 veröffentlicht.

22 Das Manuskript von 1803 und die Vorlesungen am Collège de France Nr. 1 bis 35 befinden sich in der Bibliothek der Faculté de Médecine de Paris. Die restlichen 126 Vorlesungen sind in der Bibliothek der Faculté de Médecine de Nantes. Ich möchte beiden Institutionen auch hier meinen wärmsten Dank dafür aussprechen, daß sie mir gestattet haben, diese Manuskripte zu photographieren. 
zwischen den Ungewißheiten der Empiriker und dem Fanatismus der Ätiologen den Weg des Hippokrates gehe. «Les faits constituent seuls la science» (AG I : XV). Diese Tradition sei auch von jeher auf diesem Lehrstuhl von Duret und Goupil bis Corvisart und Hallé vertreten worden. Lænnec war zweifellos überzeugt, daß er nur die Hippokratische Tradition fortsetze. Sein Freund Cayol sah darin klarer, indem er in ihm einen der Mitgründer einer neuen anatomischen Medizin erblickte ${ }^{23}$.

Krankheiten sind, so fährt Lænnec in der zweiten Vorlesung fort, Veränderungen der festen oder der flüssigen Bestandteile des Körpers oder des «principe vital». Er bezweifelt, daß es primäre Veränderungen der festen Bestandteile gibt, will aber doch in dieser Frage Eklektiker sein. Die nächsten 26 Vorlesungen bieten dann unter dem Titel «Pathologische Veränderungen der festen Bestandteile» die allgemeine pathologische Anatomie.

Das durch die Entzündungsmanie von Broussais so wichtige Kapitel der postmortalen Veränderungen wird zuerst abgehandelt. Hypertrophie, Atrophie, Kongestion folgen. Als Ursache der letzteren versucht Lænnec «Konzentration und Expansion» an Stelle der «Irritation» zu setzen und bringt die Epilepsie als Beispiel. Die Entzündung definiert er als «congestion sanguine avec tendance suppurative». Sie ist ein Ausdruck der natura medicatrix, die aber ihr Ziel überschreiten kann.

Die «Productions accidentelles» (dieser Baylesche Terminus wird von uns mit «Neubildungen» übersetzt) werden dann (Vorlesungen 10-18) im Sinne Bichats nach Geweben geordnet von den Neubildungen seröser Membranen bis zu denen der Fettgewebe dargestellt. Echte Transformationen eines Gewebes in ein anderes sind seltener, als man im allgemeinen denkt. Dies richtet sich vor allem gegen Dupurtren und Cruveilhier, die häufig zitiert werden.

In den Vorlesungen über nichtanaloge Neubildungen (19-23) werden nun bereits acht derartige Formen, d.h. Tuberkel, Encephaloide, Melanosen, kolloider Squirrhus (zum Teil unser Osteosarkom), Sklerose (eine weitere Krebsform), Cirrhose, squamöser Squirrhus und Mischgeschwülste beschrieben. Die belebten Fremdkörper, zu denen auch die von Lænnec vielstudierten Würmer gehören, beanspruchen fünf Vorlesungen (24-28).

Vorlesungen 29 bis 68 sind den Allgemeinerkrankungen geweiht. Selbst Lokalerkrankungen - Lænnec bezweifelt später, ob solche überhaupt existieren - beeinflussen den Gesamthaushalt des Körpers entweder direkt oder

23 J.B. Cayol, Clinique Médicale, Paris 1833. 
durch Sympathie (nach ihm ein viel mißbrauchter x-artiger Begriff) und führen so zu Allgemeinerkrankungen. Umgekehrt sind letztere oft von Lokalerkrankungen gefolgt.

Lænnec beginnt die Diskussion der Allgemeinerkrankungen mit den Fiebern, bei denen er die symptomatischen und idiopathischen (essentiellen) unterscheidet. Nach einer historischen Übersicht der verschiedenen Fieberklassifikationen wendet er sich den Veränderungen in Organen und Körperflüssigkeiten bei Fiebern zu. Man kann eine lokale Läsion nur als ursächlich anerkennen, wenn sie konstant gleichzeitig mit oder vor dem Fieber auftritt und genügend schwer ist ${ }^{24}$. Die leichten Veränderungen bei Fiebern in Herz, Hirn, Lunge usw. sind Folgeerscheinungen. Veränderungen werden auch gefunden in Blut, Lymphe, ausgeschiedenen Flüssigkeiten und Galle. Die Rolle der letzteren hat man vielleicht früher überschätzt, jetzt vergißt man sie zu oft. Die Symptome der obenerwähnten Läsionen werden aufgeführt.

Beim Verlauf der Fieber spricht Lænnec ausführlich über Krise, Coctio und kritische Tage (34. und 35. Vorlesung), «Doktrinen», denen er rückhaltlos anhängt, im letzteren Fall sogar gegen seinen Lehrer Corvisart. Nach Darstellung der Prognose und der Behandlung der Fieber geht er zu den einzelnen Fiebern über: den intermittierenden, remittierenden, perniciösen, symptomatischen, eruptiven ${ }^{25}$. Bei der Pest bleibt er, entgegen der Zeitmode, Kontagionist ${ }^{26}$. Erysipel, Zona, Pemphigus, Aphthen und Nesselfieber schließen die Abteilung Fieber.

An anderen Allgemeinerkrankungen werden behandelt: Skorbut, Schlangenbiß, andere Gifte, Tollwut, die im Gegensatz zu Griechenland weitverbreitete Hypochondrie (er bringt seinen Vater als Beispiel), Melancholie, Syphilis, Gonorrhoe, Gicht, Rheumatismus und Neuralgien. Lænnec schließt die Allgemeinerkrankungen mit den Diathesen ab, die erworben oder angeboren sein können. Er unterscheidet die lymphatische (aus der Skrofeln, wie Rachitis, erwachsen können), die entzündliche, die hämorrhagische, die septische sowie Spezialdiathesen, besonders bei Neubildungen, wie die tuberkulöse und die krebsige.

${ }^{24}$ In der 1.Vorlesung des zweiten Jahres fügt er als Kriterien hinzu: Gleichzeitig darf keine andere, schwere Läsion existieren. Dieselbe Läsion muß bei andern nicht ganz andere Symptome produzieren.

25 Für letztere s. auch B. Bianchetti, Charles Billard und sein Traité des maladies des enfants (1800-32), Zürich 1963, S. 41.

26 E. H.ACKerknecht, Anticontagionism 1821-67, Bull. Hist. Med. 22 (1948) 562. 
Die Behandlung der Lokalerkrankungen beginnt mit denen des Gehirns, seiner Häute und des Rückenmarks (Vorlesungen 70-81). Dann kommen neun Vorlesungen über «Neurosen» (Vorlesung 82 des ersten Jahres, Vorlesungen 1-8 des zweiten Jahres), welche hauptsächlich die Geisteskrankheiten umfassen und über die ich schon an anderer Stelle berichtet habe ${ }^{27}$. Lænnec folgt hier im Klinischen PineL, steht aber grundsätzlich auf dem «romantischen» Standpunkt, daß die Geisteskrankheiten eigentlich eher Sache des «Philosophen und Moralisten» (Theologen?) als des Mediziners seien.

Mit Tetanus und sechs Gruppen von Nervengiften kehrt er dann wieder zu den organischen Nervenkrankheiten zurück. Besonders eingehend beschäftigt er sich mit der «rachialgie» welche im wesentlichen der Bleivergiftung entspricht. Vorlesungen 15 bis 24 bringen die Krankheiten von Ohren, Augen, Gesicht, Nase und Hals zur Sprache. In Vorlesung 24 wird auch die «angine couenneuse» de Bretonneau et Guersent behandelt ${ }^{28}$. Vorlesungen 25-43 bringen die Krankheiten der Lunge in derselben Art wie der «Traité». Von der Entdeckung der mittelbaren Auskultation bemerkt Lænnec, daß sie zufällig war. «Im allgemeinen sind uns die wirklich nützlichen Dinge alle so gegeben und nicht durch Anstrengungen erobert worden.» Mit Vorlesung 44 und 45 beginnen die Herzkrankheiten, die wohl auch der Gegenstand der fehlenden Vorlesungen 46 bis 54 waren.

Vorlesungen 54 bis 76 beschäftigen sich mit den Erkrankungen des Verdauungsapparats. Die Zungenveränderungen werden ausführlich dargestellt, aber diese griechisch-chinesische Lehre als reformbedürftig bezeichnet. Die Behandlung der Gastritis und ihrer «Sympathien» bringt erneut eine Auseinandersetzung mit Broussais. «Rien n'est moins prouvé malgré l'ancienneté de l'opinion que l'inflammation des organes abdominaux dans la production de ces affections générales ou cérébrales» (Fieber). Pancreastumoren hat Lænnec ziemlich häufig gesehen. Bei der Leber vermutet er mehrere Funktionen außer der Gallenbildung (Blutbildung, Modifikation des durch die v. portae einströmenden Bluts).

Vorlesungen 77 bis 83 sind den harnbereitenden Organen gewidmet. Die neuen Lithotriptoren ${ }^{29}$ werden berichtet und sogar gezeichnet. Vorlesungen 83-87 bringen die Erkrankungen der Geschlechtsorgane, 88 und 89

27 Gesnerus 19 (1962) 93.

28 Úber Bretonneau s. P.TriaIRe, Bretonneau et ses correspondants, Paris 1892.

29 A. Gadient, Die Urologie in Paris 1800-1850, Zürich 1963. 
Hautkrankheiten. Er folgt hier Alibert ${ }^{30}$. Die letzte Vorlesung enthält einen Abschnitt über spontane Verbrennung, an die ja bekanntlich Dickens und ZouA noch glaubten ${ }^{31}$.

- Die Collège-de-France-Vorlesung, imposant in ihrer vielseitigen Kompetenz, bringt nichts grundsätzlich Neues. Immerhin treten durch sie einige Züge im Bilde Lænnecs sehr viel deutlicher hervor. Seine feste Verwurzelung in der Pathologischen Anatomie bestätigt sich wieder. Im Gegensatz zum fast nur Eigenes berichtenden «Traité» zeigt Lænnec sich hier als sehr gelehrt und zitiert eine außerordentliche Menge alter und zeitgenössischer Autoren.

Und er zitiert die Alten nicht nur. Mit seinen Auffassungen über das Primat der generalisierenden Humoralpathologie, die epidemischen Konstitutionen, die galligen Krankheiten, die Allgemeinsymptome und Krisen erweist er sich als außerordentlich traditionsgebunden und «klassizistisch», mehr noch eigentlich als seine Lehrer. Er lehrte in der Klinik auch bezeichnenderweise in lateinischer Sprache. Diese Einstellung erklärt dann wieder teilweise, warum der doch viel unbedeutendere, aber radikal mit der Vergangenheit brechende Broussais ihm von so vielen vorgezogen wurde. Diesen seinen Glauben an eine Wissenschaft, deren größerer Teil bekannt und unveränderlich ist, können wir heute kaum mehr nachfühlen.

Lænnecs Traditionalismus entspricht durchaus seinen politisch-religiösen Auffassungen, aber ganz und gar nicht seinem eigentlichen Beitrag zur Medizin. Hat er doch die Hauptfundamente eines neuen Lokalismus und Solidismus geliefert und das Hauptinstrument zur Entwicklung einer Lokalisationpathologie erfunden. Man muß ihn darum wohl als einen Neuerer wider Willen bezeichnen. Dies ist nicht der einzige Fall in der Medizingeschichte, wo ein sonst durchaus konservativer Mensch von seinem Dämon zur revolutionären wissenschaftlichen Neuschöpfung getrieben wird. Auch Thomas Willis ${ }^{32}$ oder SchwanN ${ }^{33}$ verkörpern diesen eigenartigen, spannungsreichen Typ.

Sehr ausgesprochen und dem Traditionalismus Lænnecs verwandt sind in den Vorlesungen auch sein Antimikroskopismus und sein Glaube an die

30 F.EBneter, Die Dermatologie in Paris 1800-1850, Zürich 1963. Über Alibert s. die Biographien von A. Alfaric, Paris 1917, und L. Brodier, Paris 1923.

31 J.R.Oliver, Spontaneous Combustion, Bull. Hist. Med. 4 (1936) 559.

32 H.R.Isler, Thomas Willis, Stuttgart 1963.

${ }^{33}$ M. Florkin, Naissance et Déviation de la Théorie Cellulaire dans l'œuvre de Theodore Schwann, Paris 1960. 
Heilkraft der Natur, welcher übrigens nicht eine sehr vernünftige Malariaund Skorbutbehandlung und Versuche mit neuen Medikamenten (Jod, As) ausschließt. In der Vorlesung erweist Lænnec sich als wesentlich aggressiverer Polemiker als in den veröffentlichten Schriften.

Hätte Lænnec länger gelebt, so wäre aus diesen Vorlesungen zweifellos das Buch erwachsen, das schon sein Lehrer Corvisart geplant - und auch nicht geschrieben hatte: «De sedibus et causis morborum per signa diagnostica investigatis et per anatomen confirmatis. ${ }^{34}$ Sein Manuskript steht nicht nur weit über den grob empirischen Produkten seiner Landsleute Lieutaud (1767) und Portal (1803), nicht nur über den sehr bedeutenden (erst 1825 veröffentlichten) Vorlesungsnotizen von BICHAT zur pathologischen Anatomie (1801), der Médecine éclairée par l'observation et l'ouverture des corps des Bichat-Bayle-Schülers P.A.Prost (1804) oder den Phlegmasien des Bichat-Schülers Broussais (1808), sondern auch über dem 1816 erschienenen Essay des Dupuytren-Schülers Cruveilhier ${ }^{35}$, ja sogar LoвSTEINs Werk von 1833. Nur ANDrAL, der also nicht nur Broussais, sondern auch Lænnec «aufhob», gelang es in seinem Traité d'Anatomie Pathologique von 1829 mit den von Lænnec selbst geschaffenen Methoden, über ihn hinauszukommen. Das Erscheinen dieses Werks hat wohl vor allem den Neffen und Mitarbeiter Meriadec Laennec veranlaßt, von einer begonnenen Überarbeitung und Veröffentlichung des Manuskripts Abstand zu nehmen.

So ist Lænnecs letztes, umfassendstes Werk nie erschienen. Ein Teil der Krankheiten, die er beschrieb, sind fast aus unserm Gesichtskreis verschwunden. Aber noch immer ist das von ihm erfundene Instrument das Symbol unseres Standes und das, was er in den wenigen Jahrzehnten seines kurzen Daseins unter den ungünstigsten Verhältnissen geschaffen; das, was er war, ist so gewaltig, daß er unvergessen sein wird, solange es eine Medizingeschichte gibt.

34 L. Héchemann, Corvisart, Paris 1900, S. 56. Siehe auch P.Ganière, Corvisart, Paris 1951.

35 Dieser weist mit Recht auf M.BAILLIES bahnbrechendes Buch von 1793 hin. Über Cruveilhier s. L. Delnoume, Jean Cruveilhier, Paris 1937. 